\title{
Exploring the Potential to Uniquely Manufacture Curved VARTM Epoxy Composites Using Cost-Effective FDM Molds
}

\author{
Syed Monis Raza Kazmi*, Jens Schuster, Johannes Lutz \\ Hochschule Kaiserslautern, University of Applied Sciences, Pirmasens, Germany \\ Email: ^skaz012@aucklanduni.ac.nz, jens.schuster@hs-kl.de
}

How to cite this paper: Kazmi, S.M.R., Schuster, J. and Lutz, J. (2020) Exploring the Potential to Uniquely Manufacture Curved VARTM Epoxy Composites Using Cost-Effective FDM Molds. Open Journal of Composite Materials, 10, 45-65.

https://doi.org/10.4236/ojcm.2020.103004

Received: March 30, 2020

Accepted: June 16, 2020

Published: June 19, 2020

Copyright $\odot 2020$ by author(s) and Scientific Research Publishing Inc. This work is licensed under the Creative Commons Attribution International License (CC BY 4.0).

http://creativecommons.org/licenses/by/4.0/

\begin{abstract}
The Resin Infusion or the VARTM (Vacuum Assisted Resin Transfer Molding) process has significant potential to be used to manufacture curved composites. Another way to produce curved or complex geometry is to use $3 \mathrm{D}$ printers. 3D or FDM (Fused Deposition Modelling) printers are now being used to produce relatively cheaper curved parts using thermoplastics such as PLA. However, the strength and mechanical performance of these parts is limited and can be enhanced if the polymer is reinforced with a type of fiber for instance. Research is being carried out to produce fiber rein-forced thermoplastic composites but that process is expected to be more expensive than the alternative methods such as injection or compression molding. Furthermore, to understand the manufacture of a hybrid composite using thermoplastics, fibers and epoxy resin, research and investigation need to be carried out. In this research, there are single-sided, double-sided, reusable, disposable and consumable molds. Most of the molds were created either using an FDM printer or manually. These molds were then used to manufacture flat and curved composite structures via the resin injection process, i.e. VARTM with epoxy resin system and glass/carbon/flax fiber reinforcement. By replacing the costly metallic molds by significantly cheaper molds, the cost of production was expected to further reduce. Furthermore, using double-sided PLA molds was not expected to be a threat to the overall cost of the composite part in question compared to double-sided matched molds used in compression molding. Shear strength, tensile strength and charpy impact strength of most of the manufactured composite parts were also investigated. The strengths were compared based on the method of mold usage. The results showed that this method is effective for a cheaper production of curved epoxy resin composites. However, the strength of the part will decrease as the curved profile gets more complicated unless the basic resin infusion process is altered.
\end{abstract}




\section{Keywords}

3D Printing, FDM, VARTM, Curved Composites, 3D Molds, Reusable Molds, Consumable Molds, Disposable Molds

\section{Introduction}

The Resin Infusion (aka. Vacuum Assisted Resin Transfer Molding VARTM) process has been developed over the last twenty years to manufacture large-scale composite parts for commercial and military applications. This technique is being increasingly used to produce parts and tools in the marine, wind energy, automotive and aerospace sectors [1] [2], being particularly suitable for medium to large components, and low production runs [3]. VARTM is a cheaper technique if compared to other sealed mould, compression moulding or autoclave manufacturing techniques, especially as part size increases. When compared to the wet hand lay-up process and Resin Transfer Molding, it minimizes styrene and various gaseous emissions while maintaining simple single-sided mould setup. Significant amount of research is being carried out to manufacture complex shaped composites using resin infusion and test the composite parts.

Fused deposition modelling (FDM) is a particular Layered Manufacturing (LM) technique in which each section is fabricated through vector style deposition of building blocks, called roads, which are then stacked layer by layer to fabricate the final object. The $3 \mathrm{D}$ printer used in this research uses the same concept. Anna Bellini [4] laid emphasis on improving the liquefier dynamics of FDM because this technology is believed to bring about the possibility of fabricating not only a model but even the finished product or a mold for use in further manufacturing. Zein and co-workers [5] constructed Porous FDM scaffolds by forming layers of directionally aligned microfilaments within a geometrical 3D structure. They concluded that if the scaffold porosity is controlled, the scaffolds can withstand high compressive pressures. Ahm and co-workers [6] produced 3-D parts using FDM and the tensile and compressive strengths of the parts were comparable to the parts produced by injection molding with the same material. Montero and co-workers [7] supported this. Ang and co-workers [8] were in agreement with Zein and found a logarithmic relationship between the mechanical properties and the porosity of the scaffolds. Bellini and Gucery [9] also found out that the build strategy of the 3-D part or the scaffold has direct effect on the performance of the part. Bagsik and Shoppner [10] found out that the build strategy and the part structure affect the tensile strength of the 3-D part produced by FDM. In another research Bagsik and co-workers [11] believed that the FDM technology can produce manufacturing tools and even end-use parts. Hence, they determined the available mechanical data based on the process control and reproducibility. Sood and co-workers [12] found out that the major reason for weak strength may be attributed to distortion within or between the 
layers of the 3-D part. They also found in another research [13] that layer thickness, orientation, raster angle, raster width, raster air gap, raster interaction between layer thickness and orientation greatly affect the tensile, bending and impact strength of the 3-D components produced using FDM. Gray, Baird and Bohn [14] found that the final mechanical properties of a 3-D part can be tailored to a specific application by adjusting the lay-down pattern to increase the functionality of the part, and that these properties can be predicted by composite theory. Weinmann and co-workers [15] found that small air gap, small layer thickness, low raster orientation and the interaction between high temperature and small layer thickness yielded the greatest effect on the part strength tailored using FDM. Drummer and co-workers [16] found out that PLA was a suitable material for 3-D printed parts using FDM and that the processing temperature and the part size affect the structural integrity of the printed part.

Teoh and Hsiao [17] indicated that the spring-in angle of a curved specimen decreases as the number of curing stages increases. The spring-in effect is the reduction of a consolidated curved composite part when it is released from the mold. Their research can be used to improve the dimensional instability problem for manufacturing of curved composites. Besides this, they also stated that the curing process of a thick composite also faces the thermal management challenge due to the high exothermic reaction rate and low thermal conductivity of resin. Ho and co-workers [18] stated that poor wettability, poor bonding and degradation at the fibre/matrix interface (a hydrophilic and hydrophobic effect) and damage of the fibre during the manufacturing process are the main causes of the reduction of the composites' strength. Kuo [19] devised an economical method to fabricate epoxy-based composite mold inserts using rapid prototyping and rapid tooling technique. Kuo laid importance of producing cheap molds for different manufacturing processes. Oterkus and co-workers [20] worked on a non-destructive method to test the structural integrity of curved composites. The method was based on finite element method and the peridynamics theory. Minakuchi and co-workers [21] also developed a non-destructive life cycle monitoring system and an advanced quality assurance method for curved composite parts to improve the design and manufacturing of the composites.

Manufacturing of both epoxy and thermoplastic composites is an area that is constantly being researched. The task gets more challenging when the profile of the composite is more than just a flat surface. Much research has been carried out to manufacture curved composites [22]. There have been many applications [23] [24] requiring curved composite structures and research is required [25] in this field.

VARTM technique [3] [26] [27] [28] [29] has been widely used to manufacture epoxy composites with significantly complicated geometry. Most of which is employing one sided or flexible molds. Manufacturing curved composites with closed-loop or hollow reinforcements can be further explored because not much work has been carried out in this area. This research focuses on finding the po- 
tential to manufacture open and closed loop composites through VARTM using various types of molds and knitted fabrics. The trend in the thickness, shear strength and tensile strength across the curve of the composites is to be analysed. The VARTM manufactured structures in this research are aimed to be applicable in the aircraft, space construction and civil construction industries as also predicted by Soutis [1], Dong [29] and Naser [2].

\section{Experimental Program}

\subsection{Materials}

The fibre reinforcements used to manufacture composites with FDM Molds were carbon fiber $\left(93 \mathrm{~g} / \mathrm{m}^{2}\right)$, glass fiber $\left(280 \mathrm{~g} / \mathrm{m}^{2}\right)$ and coarse twill flax fiber (550 $\mathrm{g} / \mathrm{m}^{2}$ ). All fiber reinforcements were twill type. Figure 1 shows the three fiber reinforcements. Coarse twill flax reinforcement was chosen because of its twill architecture and because it was expected to form better doubly-curved composites as established by Kazmi and co-workers [22] [30].

The fiber reinforcements used to manufacture composites with non-FDM molds were weaved glass fiber reinforcements. All reinforcements used in this research were twill weaved with alternating $\left( \pm 45^{\circ}\right)$ weft and warp.

Figure 2 shows the weaved glass fibers to take the shape of a complicated structure with multiple hollow cylinders.

Larit epoxy resin system was used in all experiments. The ratio of the resin (L-160) and hardener (502) was 100:40 by mass. Gelling time was approximately

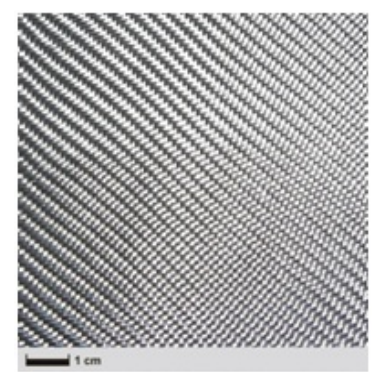

(a)

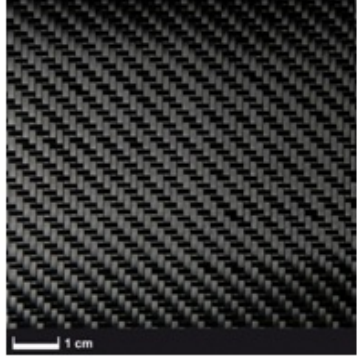

(b)

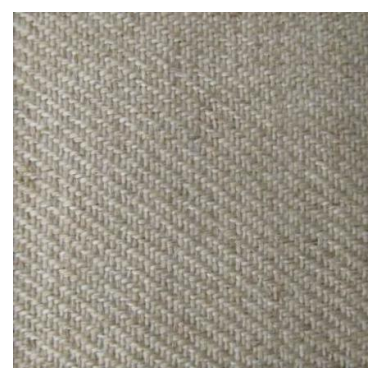

(c)

Figure 1. (a) Glass fiber, (b) Carbon fiber and (c) Flax fiber.

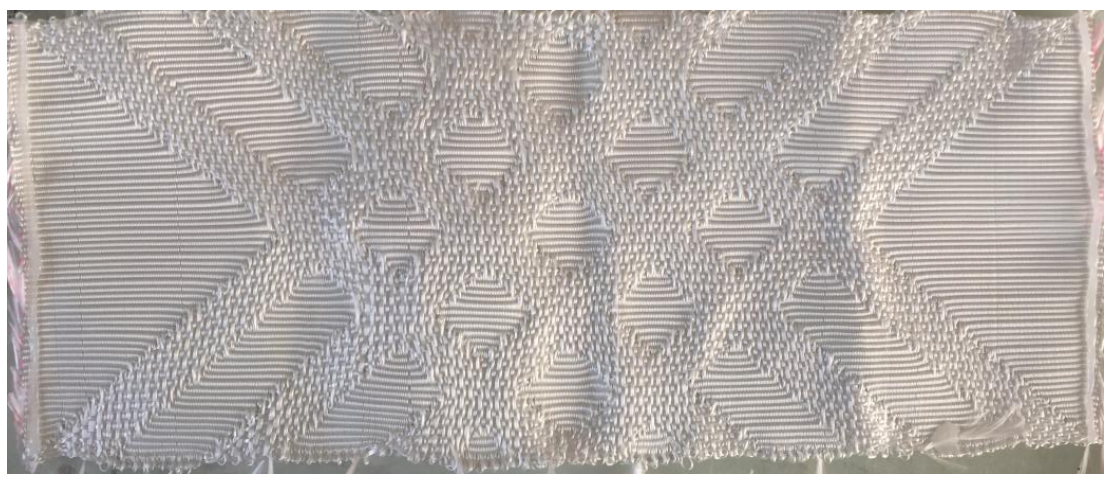

Figure 2. Closed-loop weaved glass fiber. 
50 minutes after careful mixing and degassing for 15 minutes. The epoxy resin system with a gel time of 35 minutes was chosen because of the smaller size of the molds used. A lower gel time meant that the resin would be in its liquid state for a longer period of time and that was not advised because the filling time of the mold was approximately 20 minutes.

\subsection{FDM Setup and Procedure}

An FDM printer was used to create singly-curved and doubly-curved molds. Figure 3 shows the picture and schematic of the printer. PLA was used in the form of a filament of diameter $1.75 \mathrm{~mm}$ wound on a spool as shown in Figure 3. The nozzle of diameter $0.35 \mathrm{~mm}$ was used at a temperature of $200^{\circ} \mathrm{C}$. The feed rate of liquid PLA coming out through the nozzle on the bed was $38 \mathrm{~mm} / \mathrm{s}$ and the bed was at $60^{\circ} \mathrm{C}$.

In order to print the infusion molds, the "Mendelmax FM Pro" in version 1.5 has been used. The printer in modular principle is a cost-effective option in comparison to an industrial FDM printer. In Table 1 the printer settings are displayed. A nozzle diameter of $0.35 \mathrm{~mm}$ was used to gain a reasonable printing quality. With a nozzle temperature of $200^{\circ} \mathrm{C}$, the PLA has shown proper melt behaviour and printing accuracy. Due to the staircase effect, the molds have been printed in perpendicular direction to the front side. As a result, the front side itself shows the best surface quality, the printer is able to. The solid infill ratio of $35 \%$ has been suitable to withstand the applied pressure and kept the mold from collapsing by the use of vacuum pressure. If printing time would not have been the primary issue, a higher degree of filling could have been achieved resulting in a stronger mold with a greater resistance to compaction pressures. For a higher degree of filling, the printing time will increase noticeably.
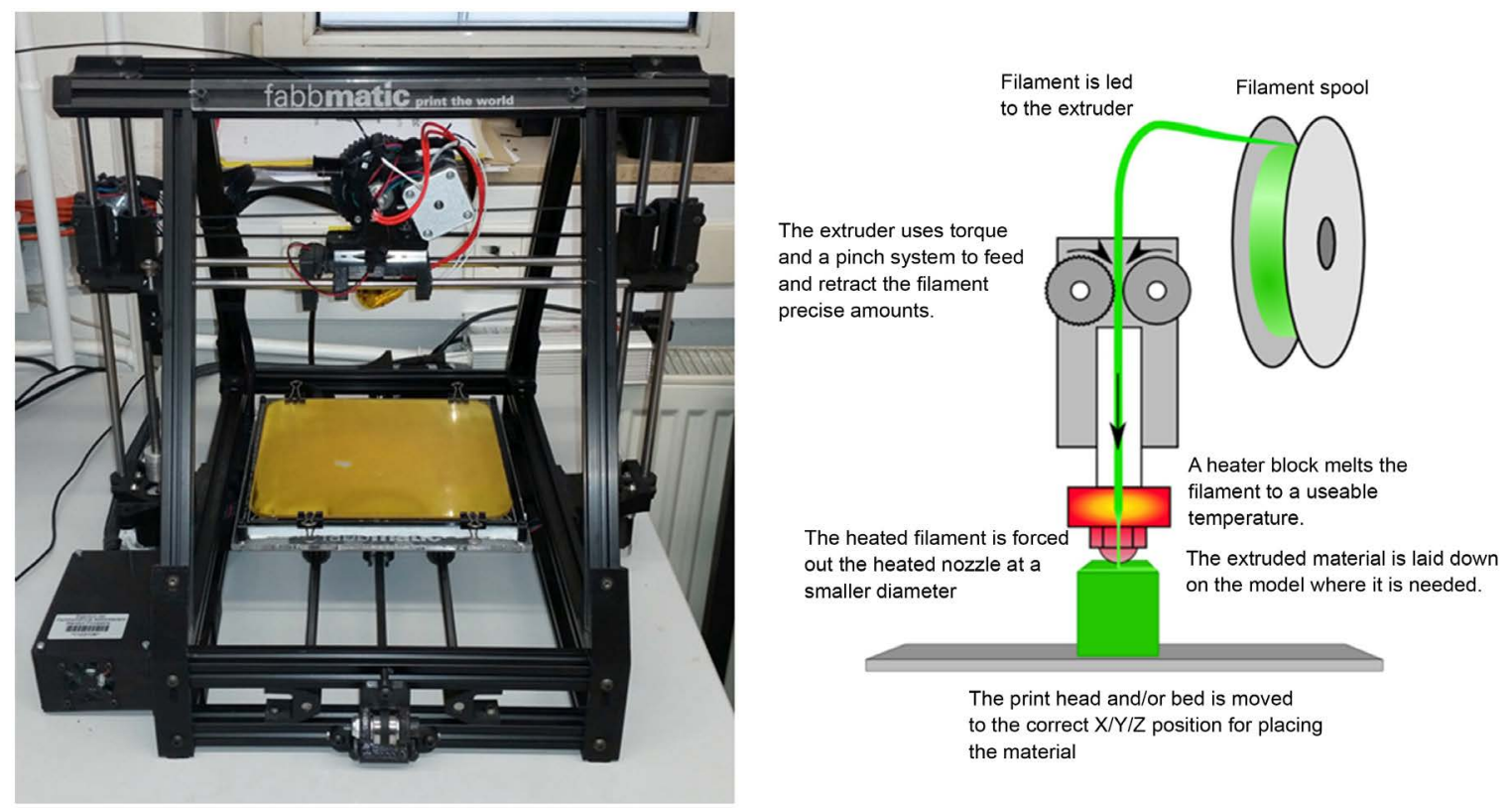

Figure 3. FDM printer and its mechanism. 
Table 1. FDM printer parameters.

\begin{tabular}{cc}
\hline Nozzle diameter & $0.35 \mathrm{~mm}$ \\
Filament diameter & $1.75 \mathrm{~mm}$ \\
Nozzle temperature & $205^{\circ} \mathrm{C} 1^{\text {st }}$ layer, $200^{\circ} \mathrm{C}$ remaining layers \\
Bed temperature & $60^{\circ} \mathrm{C}$ first layer, $55^{\circ} \mathrm{C}$ remaining layers \\
Layer height & $0.15 \mathrm{~mm}$ \\
Feed rate & $38 \mathrm{~mm} / \mathrm{s}$ \\
Infill solidity ratio & $35 \%$ \\
Minimum detail & $0.3 \mathrm{~mm}$ \\
Minimum clearance & $0.5 \mathrm{~mm}$ \\
XY-axis positioning accuracy & $0.0015 \mathrm{~mm}$ \\
Z-axis positioning accuracy & $0.005 \mathrm{~mm}$ \\
Part Accuracy & $\pm 0.3 \%-0.5 \%$ \\
Max. part size (LxWxH) & $150 \times 150 \mathrm{~mm} \times 150 \mathrm{~mm}$ \\
\hline
\end{tabular}

\subsection{FDM Generated Molds}

The previously explained FDM printer was used to create single and double sided molds to manufacture composites with the VARTM process. The FDM molds were primarily used to compare the geometries of the VARTM epoxy composites. An infusion pressure of about 0.8 bar will be used so that the mold can withstand the pressure of the applied compaction force. Negative and positive molds were produced to "muffle" the reinforcement material. It was expected to achieve a better surface finish with the double-sided mold upgrading the idea of Professor Summerscales [31], who suggested to use a flexible vacuum bag instead of a double-sided mold. He was referring to the costly metallic molds but in this case, significantly cheaper PLA molds were used. Hence, using double-sided PLA molds will not be a threat to the overall cost of the composite part in question.

Figure 4 shows the male and female parts of the singly and doubly curved composites produced by the FDM Printer. The female singly-curved mold had a cavity which was a semi-cylinder of radius $25 \mathrm{~mm}$ and length of $100 \mathrm{~mm}$. The cavity for the doubly-curved mold was a hemisphere of radius $25 \mathrm{~mm}$.

\subsection{Manually Prepared Molds}

The manually prepared molds were created to manufacture the hollow cylindrical composites and the structure with multiple hollow cylinders. These molds were created to induce the best possible cylindrical shape and surface finish in the manufactured composites and this was one of the most challenging parts of this research. The reason for this was the de-molding process. The outer surface of the mold was made soft enough to remove the cylindrical composite after manufacturing but hard enough so that the composite could take a good cylindrical shape and surface finish. Readily available PVC pipes were used and rubber tape 

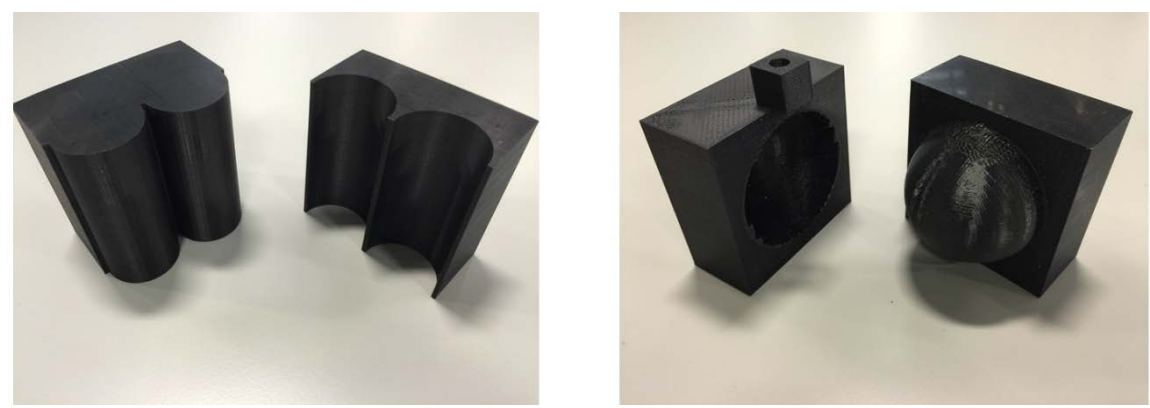

Figure 4. Singly curved and doubly-curved PLA molds created by the FDM printer.

was used on the pipes to create the required diameter so that the closed loop weaved glass fiber reinforcements can fit on and removed from the pipes conveniently. For the special structure with the hollow cylinders (Figure 2) smaller PVC pipes were fitted inside the holes for the flat structure and flexible rubber pipes were fitted for the curved structure.

\subsection{VARTM Setup and Procedure}

Figure 5 shows the schematic of experimental setup employed for the resin infusion (VARTM) process. The fiber reinforcement was kept inside the single-sided or double-sided mold. The mold was covered with a nylon bag so that it could be reused and the manufactured composite part could be easily removed after the experiment. A finely perforated fabric known as peel ply was used to create vacuum inside the mold by removing the air inside the cavity through a polyethylene port as shown in Figure 5. The vent catch pot was used in case any excess resin was drawn out of the mold, to avoid any damage to the vacuum pump. Moreover, just before the port connected to the vent catch-pot, cotton was inserted between the mold and the vent port to decrease the filling speed of the resin and to block the resin from going to the vent catch-pot or the pump. When the nylon bag was vacuum taped on glass, the vacuum pump was turned on. When all the air inside the cavity was extracted through the vent catch pot, vacuum was created and this vacuum drove the resin from the inlet pot through the connecting tube into the mold cavity. When the resin filled all the coarse twill reinforcement it was blocked by the cotton. After the resin solidified, the vacuum pump was left turned on overnight and the next day the de-molding of the composites was followed by its post-curing. The standard industrial VARTM process was followed where first the resin fills the mold and when the resin hits the break, the inlet is clamped and left overnight for curing. During curing overnight, the vacuum pump keeps running and the vent stays at full vacuum which is around 900 mbars of compaction pressure in this case.

\subsection{Experimental Plan and Measurements}

This research aims to explore the potential of VARTM to manufacture curved composites using cost-effective molds. Three types of molds were manufactured using the FDM printer. The structural integrity of the flat composites was tested 


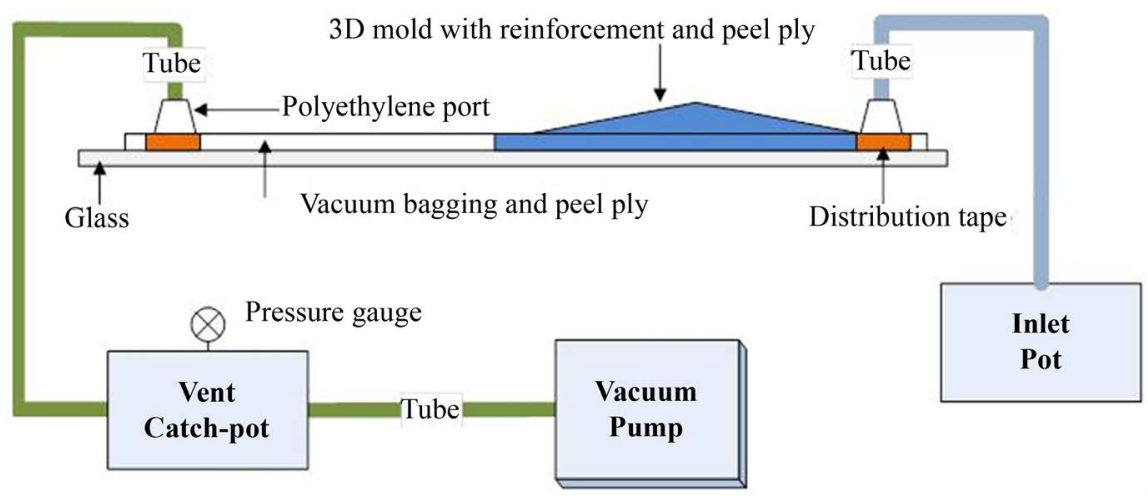

Figure 5. Schematic of the experimental setup employed for the VARTM process.

by using tensile, bending, and charpy testing. Table 2 presents the manufacturing plan for manufacturing and testing of the composites. Manufacturing was aimed to start from flat to simpler curvatures and then to the more complicated structures. All experiments were repeated to cross check the results. Number of layers of the fibers infused with resin was varied to keep the areal mass of the fiber reinforcements constant at $300 \mathrm{~g} / \mathrm{cm}^{2}$. All the manufacturing details will be kept constant for repeatable results. Bending tests were carried out to show the structural integrity of the curved composite parts. Thickness at different positions of the curved composites was measured to determine the dimensional consistency of the composite parts.

\subsection{Molding of Closed Loop Composites}

The hollow composites needed special molding technique because of having a closed loop. There were two challenges. The first was to create the desired hollow cylindrical shape and the second was to de-mold the composite from the cylindrical mold. Figure 6(a) shows how a cylindrical mold was used with other consumables and the weaved glass fiber reinforcement before resin was infused through the assembly. First, a soft rubber core (vacuum tape) was wrapped around the solid cylindrical mold. This was then covered by a vacuum bag sealed by a vacuum tape in order to create vacuum. The glass fiber reinforcement covered from both sides by a breather (peel ply) was then rolled over the vacuum bag. A distribution medium was wrapped around the breather for a faster flow of the resin through the reinforcement. In the end everything was sealed using a vacuum bag and vacuum tape.

Two types of structure with multiple hollow cylinder network were manufactured. One was flat and the other curved. Figure 6(b) shows how the flat structure was prepared before resin infusion. Multiple PVC hollow pipes were used as shown so that the structure could be formed. For the parts were the pipes could not reach, smaller sections were cut and placed but those sections were not expected to come out of the composite structure. After placing all the hollow pipes inside the appropriate place in the weaved glass fiber reinforcement as shown in Figure 6(b), the reinforcement was wrapped by a breather (peel ply). A distribution 
Table 2. Plan of manufacturing and testing of composites.

\begin{tabular}{|c|c|c|c|c|}
\hline & Type of Composite & Mold Type & $\begin{array}{l}\text { Number of } \\
\text { Composites }\end{array}$ & Type of Test \\
\hline 1 & Flat weaved & Flat Plate & 3 & Tensile and Short Beam Shear \\
\hline 2 & Open Loop Simple curvature & & 3 & \\
\hline 3 & Open Loop Singly curved & $\begin{array}{l}\text { Single and Double } \\
\text { Sided FDM Molds }\end{array}$ & 3 & $\begin{array}{l}\text { Short Beam Shear and } \\
\text { Thickness Measurements }\end{array}$ \\
\hline 4 & Open Loop Doubly curved & & 3 & \\
\hline 5 & Closed Loop Hollow cylinder & & 3 & Tensile and Short Beam Shear \\
\hline 6 & $\begin{array}{l}\text { Complicated Closed Loop } \\
\text { Flat multiple hollow cylinder } \\
\text { assembly }\end{array}$ & $\begin{array}{c}\text { Manually } \\
\text { Prepared Molds }\end{array}$ & 3 & None \\
\hline
\end{tabular}

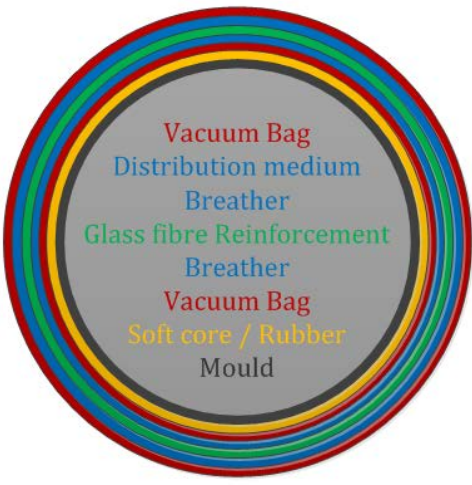

(a)

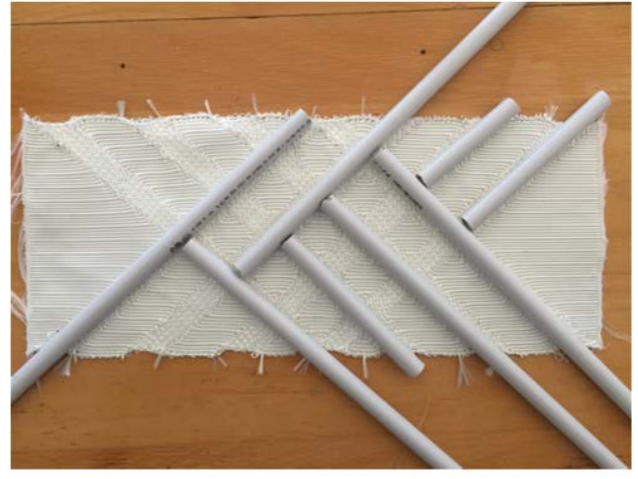

(b)

Figure 6. Molding technique for the composites (a) Hollow cylinder and (b) Flat web.

medium was placed on the reinforcement and then everything was vacuum bagged and connected to the inlet and vent for VARTM (Figure 5).

For the curved structure, instead of the solid PVC pipes, flexible rubber pipes were used but in this case most of the rubber pipes stayed inside the composite because there was no way to bring them out after infusion. The curved structure was placed on a curved surface to achieve the required angle and then the whole mold was infused with resin.

\subsection{Thickness Measurements}

Coarse twill flax reinforcement was used with epoxy resin. Four singly-curved and four doubly-curved composites were manufactured. Two composites each were manufactured using the single-sided female molds and two composites each were manufactured using double-sided singly and doubly-curved molds shown in Figure 4. Three layers of coarse twill reinforcement were used to manufacture all the composites. The cutting of the reinforcement before manufacturing the doubly-curved composites was executed as described in by Kazmi and co-workers [22]. Thickness of both the singly-curved and doubly-curved composites was measured at five different points. A total of five specimens were cut from each composite part to be used for measuring their thickness and testing 
them under shear. These measurements were executed for all the composites. The choice of these points was made to find the trend of thickness and shear strength across the curve for both the singly and doubly-curved composites. Figure 7 shows the positions where the thickness of the singly and doubly-curved composites were measured. These are also positions from where the specimens for the shear tests were obtained.

\subsection{Destructive Testing}

Short beam shear and tensile tests were conducted on samples to evaluate the effect of variation in the fiber and curvature on the mechanical strength of the composites. Samples taken from parts manufactured according to the manufacturing plan were tested for short beam shear strength under ASTM-D2344 standard and for tensile strength under ASTM-3039. A total of five specimens were cut out of each composite part to be used for testing. The specimens were cut out from the manufactured composite parts. Instron 5567 testing machines were used to perform the bending test. The cross-head speed was set to $1 \mathrm{~mm} /$ minute to prevent abrupt failures. The criterion for specimen failure was a flexural deformation of $8 \%$. All other measurements were according to the standards.

\section{Results and Discussions}

\subsection{Open Loop Composites}

This research also aimed to compare the manufacturing of the composites using single sided PLA molds with the double sided counterparts. Single-sided and double-sided FDM molds were used so that the dimensional consistency of the VARTM manufactured epoxy composites using each mold type could be compared. Figure 8 shows the thickness across the curve of singly-curved and doubly curved coarse twill composites manufactured using both mold types. The composites manufactured using the double-sided molds were thinner than those manufactured using the single-sided molds at every position. The thickness across the curve for both composite types does not show a clear trend but the thickness range is different for the two types in Figure 8. The range is 2.77 to $2.89 \mathrm{~mm}(4.3 \%)$ for the singly-curved double-sided molded composites and 2.91 to $3.15 \mathrm{~mm}$ (7.8\%) for the single-sided counterparts. This implies that thickness
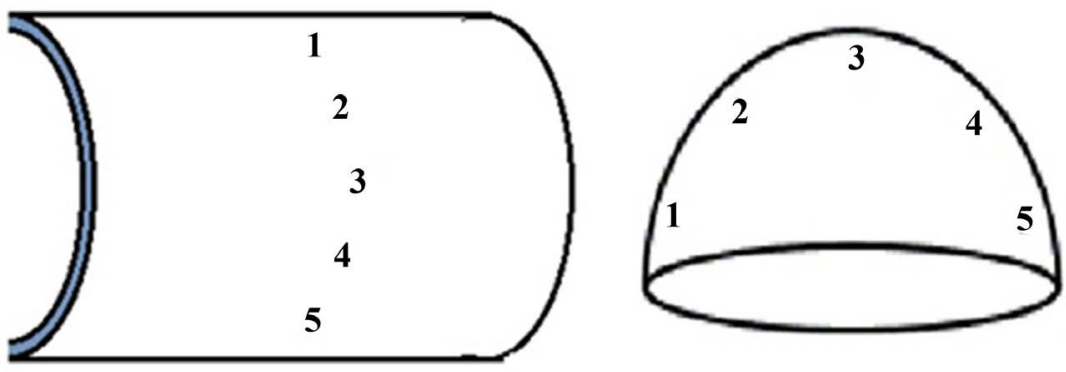

Figure 7. Positions on VARTM produced curved coarse twill composites for specimens obtained for mechanical testing and thickness measurements. 


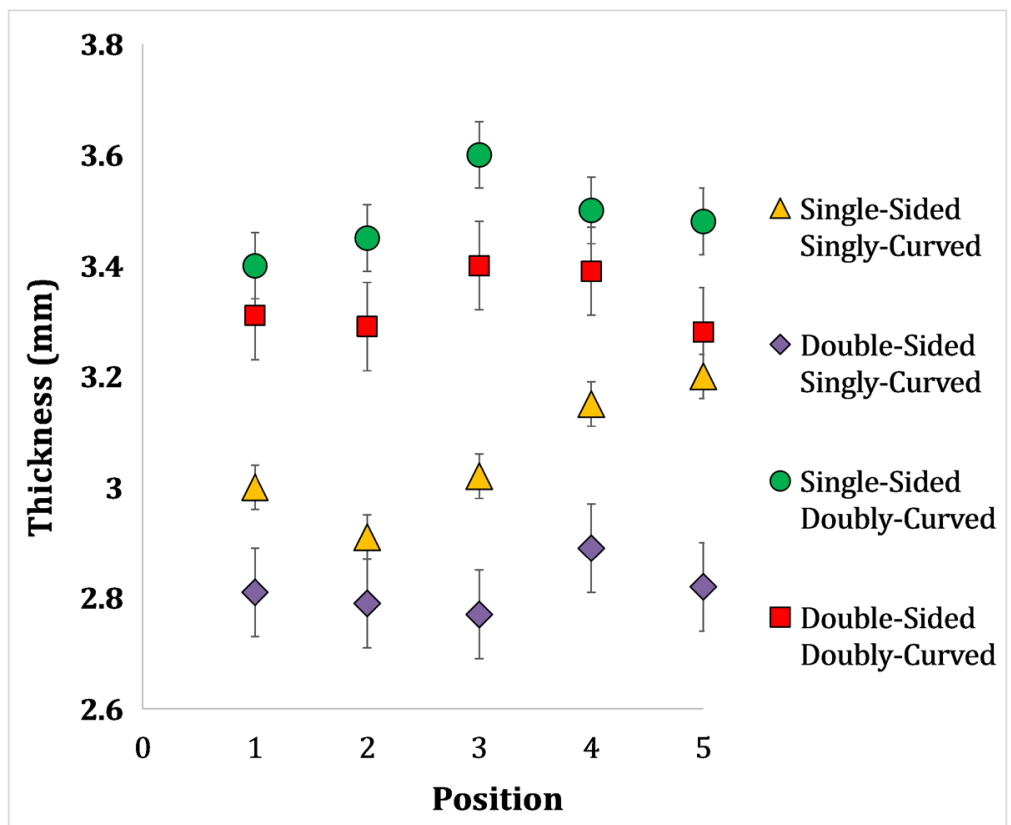

Figure 8. Across the curve thickness variation of VARTM produced singly-curved and doubly-curved composites manufactured using single-sided and double-sided FDM molds at positions shown in Figure 7.

of the singly-curved double-sided molded composites is more consistent along the curve.

Unlike the singly-curved composites, the doubly-curved counterparts show a slight trend in the thickness across the curve. Maximum thickness was recorded for both single-sided and double-sided molded composites at Position 3. As shown in Figure 7, this position is the central part of the doubly-curved composite and thickness at this point was the greatest for both composites types as shown in Figure 8. The trend is that the thickness is the least at the ends of the composite (Position 1 and 5) and maximum at the central part (Position 3). The range of thickness was 3.28 to $3.40 \mathrm{~mm}$ (3.6\%) for the double-sided molded composites and 3.40 to $3.60 \mathrm{~mm}$ (5.7\%) for the single-sided counterparts.

The parts produced with single-sided molds have a larger scatter of thickness values (7.8\% and 5.7\%) than the ones produced with the double-sided arrangement $(4.3 \%$ and $3.6 \%)$. To produce parts with a more stable thickness over the surface, double sided molds looked promising and maintained a good consistency of thickness over the surface of the manufactured parts.

The shear strengths of the singly-curved and doubly-curved coarse twill composites manufactured using single-sided and double-sided PLA molds are presented in Figure 9. The range of shear strength values across the curve of the singly-curved composites manufactured using the single-sided molds was from 17.9 to $18.2 \mathrm{MPa}$ and the range for those manufactured using the double-sided molds was from 16.6 to 17.3 MPa. The range of shear strength for the single-sided molded composites was from 10 to $10.15 \mathrm{MPa}$ and for the double-sided ones was from 9.8 to 9.9. It can be deduced that the average shear strength of both the 


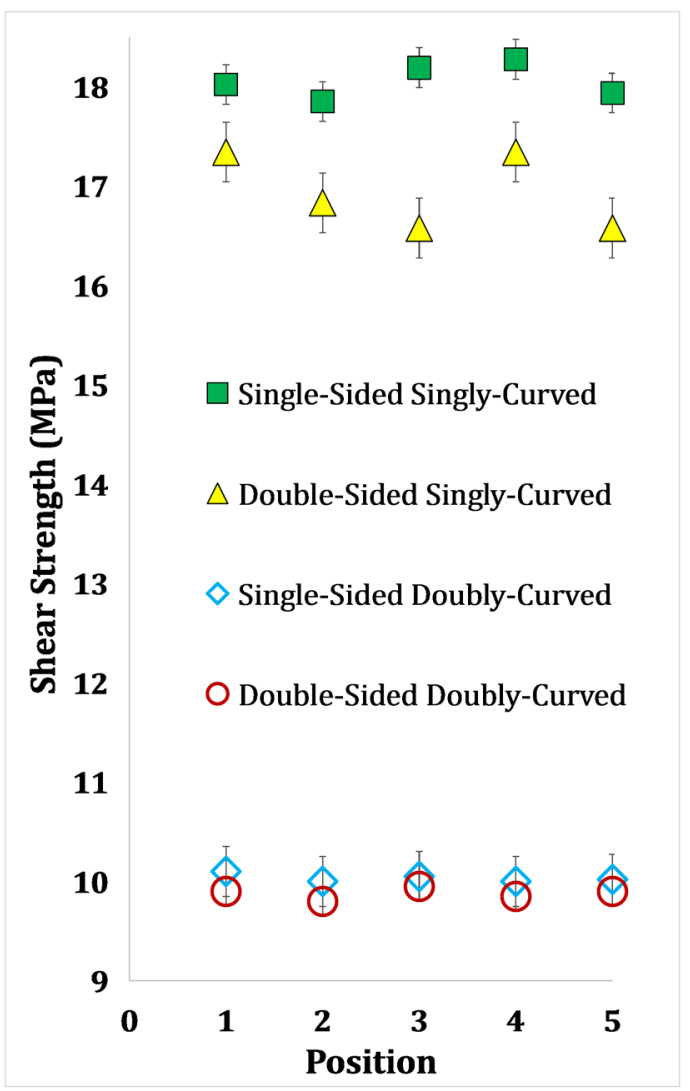

Figure 9. Across the curve shear strength variation of VARTM produced singly-curved and doubly-curved composites manufactured using single-sided and double-sided PLA molds at positions shown in Figure 7.

singly-curved and doubly-curved composites manufactured using the single-sided molds was greater than those manufactured using the double-sided molds.

Position 3 was the strongest under shear for both the singly-curved and doubly-curved composites manufactured using single-sided molds and the weakest for the double-sided mold counterparts. Considering Figure 8, Position 3 was the thickest for the single-sided molds and the thinnest for the double-sided molds. For an epoxy composite, a thinner composite is not an indication of the quality of consolidation of the composite. The thickness in the epoxy composites is only due to the compaction pressure during the infusion process and after the resin has completely filled the reinforcement. In this case, since Position 3 is the thickest part in the single-sided composite, it has more mass and hence more resistance under shear. Position 3 on the double-sided molded composite was thinnest and hence the weakest part under shear because it had a lower mass of resin.

Double-sided molds were expected to induce higher initial imperfections and voids in the parts than those manufactured using single-sided molds. The PLA molds used in this research were considered significantly weaker than any other molds being used in the industry to produce epoxy resin composites. They were 
only good enough to withstand a compaction pressure less than or equal to the atmospheric pressure, so the imperfections and voids induced by the double-sided molds were almost negligible and difficult to quantify. The low compaction pressure is the reason why the thickness of the double-sided molded composites was more consistent across the curve than the single-sided counterparts. Further research can be carried out by subjecting the double-sided molds to varying compaction pressures and then investigating the effect on the dimensional stability of the part and voids induced in it.

According to Zhu and co-workers [32], under a compaction pressure of over 6000 mbars there will be residual stress or strain in curved parts that will decrease with an increase in the thickness of the part. This phenomenon is known as spring forward in which the curved manufactured part will have a lower radius of curvature than the mold. For a $4 \mathrm{~mm}$ thick part, this strain was $1.05^{\circ} \mathrm{ac}-$ cording to the viscoelastic model and $0.69^{\circ}$ according to the elastic model. Bogetti and co-workers [33]; Miller and Ramani [34] and Ruiz and Trochu [35] established that the residual stress causing part deformation was dependent on reaction kinetics, specimen thickness and the cure cycle. Golestanian and El-Gizawy [36] found that the finished part was 1.2 to $1.38 \mathrm{~mm}$ shorter than the mold geometry over a $50 \mathrm{~cm}$ dimension.

All of these researchers used double-sided molds in their models and experiments. The compaction pressures involved were much more than the atmospheric pressure. In this research, there was no temperature gradient in the mold. The use of single-sided molds further reduced the probability of inducing residual stresses in the manufactured composites. Hence, the shear strength of the composites manufactured using the single-sided molds was better for both singly-curved and doubly-curved composites.

\subsection{Average Composite Thickness, Shear and Tensile Results}

The composites parts manufactured via the resin infusion process were tested for the shear strengths. Thickness at six points on all the parts was measured and the average thickness was determined for each type of part. Figure 10 shows the average thicknesses of all the types of glass fiber composites manufactured during this research using various types of molds. The flat composites are the thinnest because of the post-filling pressure which was not enough due to the curvature of the curved composites. This implies that the composite thickness for a part with a more complicated curve will be less compared to the one with a less complicated curve. As the curve gets complicated, it is more difficult for vacuum to press the reinforcement on the mold and hence more resin flows and impregnates the fiber reinforcement. This can be reduced by pulling vacuum from the vent and inlet in the post filling stage rather than just clamping the inlet. It will be interesting to see how close the thickness values can be brought for the given three curvatures under these conditions. On the other hand, by pulling full vacuum from both the inlet and the vent in the post-filling stage, voids will be 


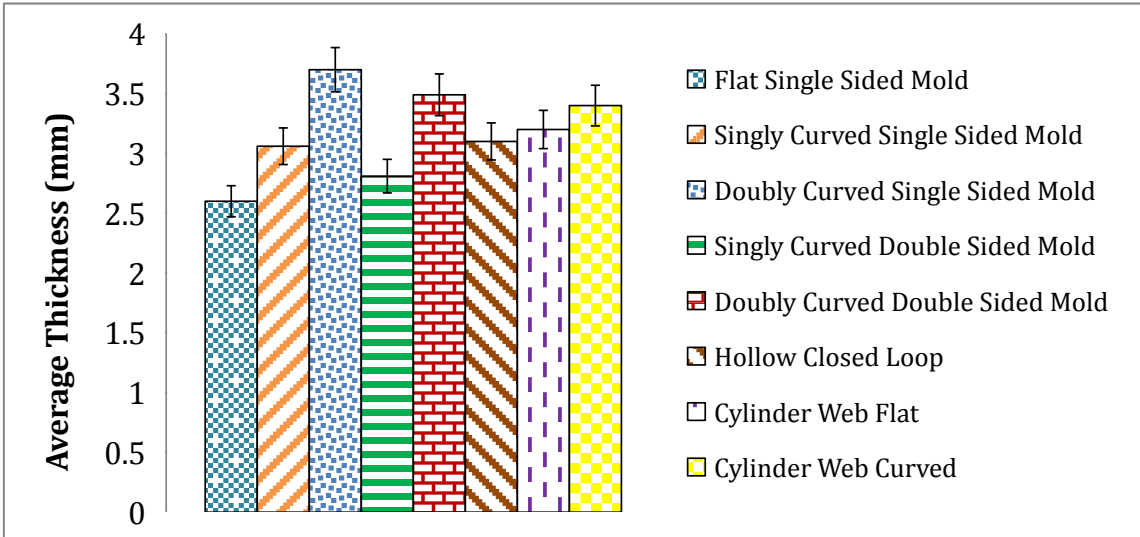

Figure 10. Average composite thickness.

induced in the composite part, which can considerably reduce the mechanical strength of the part. Hence it is a trade-off between the dimensional accuracy and the mechanical strength of the part. If a designer wants to achieve a specific type of thickness then there are many other factors including the compaction pressure to be considered. The average thickness values are only a comparison between the compressibility of each mold type keeping all the other variables constant. Also it needs to be understood that the variation in thickness will increase if the number of fiber layers are increased. Hence, the designer must know all the variables including the compaction pressure, number of fiber layers, part geometry and part size to choose a specific type of mold.

The short beam shear strengths of all the types of glass fiber composites manufactured in this research are shown in Figure 11. The flat composite is by far the strongest in shear because it is the most suitable shape for a VARTM process. Composites manufactured using single sided molds were stronger in shear than those manufactured using the double sided molds. This is mainly because there was more impregnated resin in the single sided molded composites. If a comparison is made between the different curvatures, then it is seen that there is a marked difference in the shear strengths of single and double curved composite part. This low shear strength is mainly because of the complicated curvature itself and partly because of the indirect effect of this double curve on the manufacturing process. When the part geometry gets more complicated the compaction of the fiber reinforcement due to vacuum is weaker. The hollow cylindrical composite is although weaker in shear than the flat composite but slightly stronger in shear compared to the singly curved composites. This may be because the hollow composites had a slightly larger diameter than the singly curved composites. The cylinder web composite structures were the weakest because of their complicated geometry. The obtained specimens from the webs were also not very perfect according to the ASTM standards.

When the short beam shear strengths were compared to previous research; the flat glass fiber epoxy composites were $10 \%$ stronger than those manufactured by Fan and co-workers [37] using VARTM, 61\% stronger than the woven glass fiber 


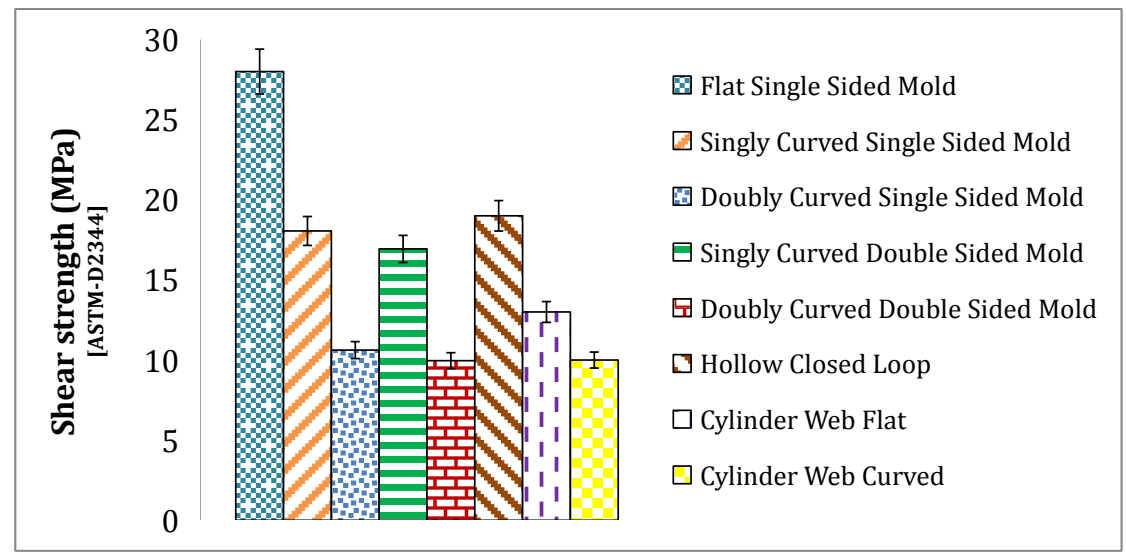

Figure 11. Average glass fiber composite short beam shear strength.

epoxy composites manufactured by Yang and co-workers [38] using RTM, 19\% stronger than the twill glass fiber epoxy composites manufactured by Almeida Jr. and co-workers [39] using VARTM and83\% stronger than the unidirectional glass fiber epoxy composites manufactured by Selmy and co-workers [40] using the wet hand layup technique. These comparisons cannot be absolute due to the difference in experimental conditions such as fiber orientation, manufacturing method and the fiber volume fraction. However, this comparison was necessary to show that the shear results of this research are promising. Most of the singly curved composites are as strong in shear as other glass fiber composites manufactured. The doubly curved composites are although weaker in shear but still comparable to the other glass fiber composites manufactured by previous researchers.

The tensile strengths of all the types of glass fiber composites manufactured in this research are shown in Figure 12. The carbon fiber flat composites were the strongest in tension while the cylinder web and singly curved composites were the weakest. The trend is very similar to the one in Figure 11. The only comparison here is between the carbon, flax and glass fibers where the carbon fiber composites were the strongest and the flax fiber composites were the weakest.

When the tensile strengths were compared to previous research; the carbon fiber epoxy composites were 5\% stronger than the ones manufactured by Godara and co-workers [41] using a vacuum autoclave. The flax epoxy composites were $25 \%$ stronger than the ones manufactured by Yan and Chouw [42] using the wet hand layup technique. The glass fiber epoxy composites were 50\% weaker than the unidirectional glass fiber composites tested by Devendra and Rangaswamy [43]. Again, since the manufacturing conditions are different, these comparisons are only to show that the tensile results of this research are also promising.

\subsection{Dimensional Accuracy and Shape}

The challenge in this research was to achieve the targeted shapes. Many experiments were unsuccessful because of the complicated process. The PLA molds used were considered significantly weaker than any other molds being used in 


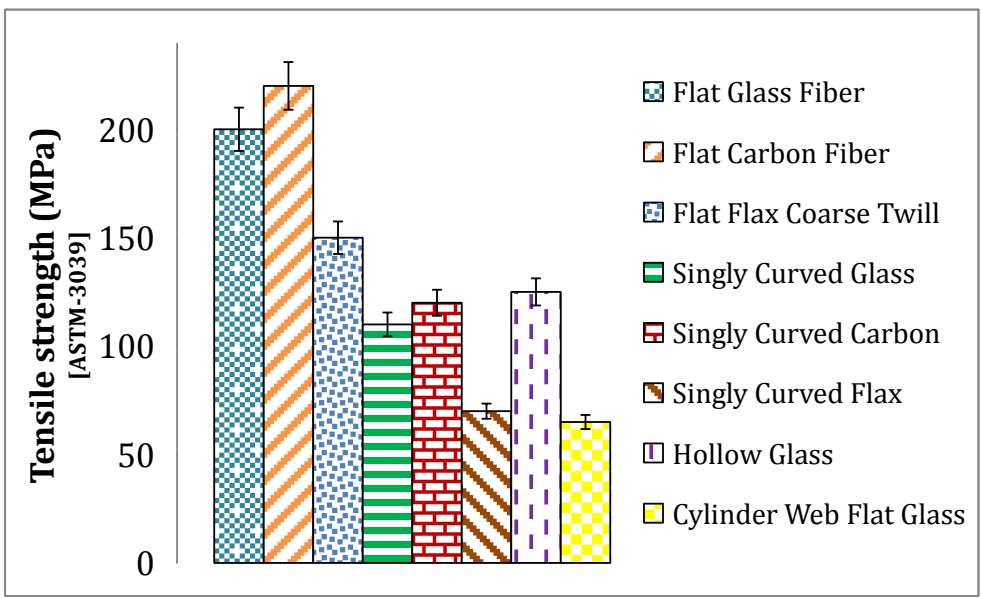

Figure 12. Average composite tensile strength.

the industry to produce epoxy resin composites. However, since the compaction pressure was not more than the atmospheric pressure, the weak PLA molds were able to withstand this stress and convert that to shape the composite part. Figure 13 shows pictures of a simple single curved carbon fiber reinforced epoxy composite part, a singly curved one and a double curved flax composite. The shape and dimensions are quite in line with what was targeted. Especially for the simple singly curved parts, the shape and dimensions were very consistent for all fiber reinforcements. The shape and dimensions for the one shown in Figure 13(b) were also quite in line with the targeted ones. Both the parts in Figure 13(a) and Figure 13(b) were manufactured using a double sided mold so that is also a reason why the shape dimensions are quite consistent. Although, parts manufactured by the single sided molds were considerably consistent in the shape and dimensional accuracy. The curve geometry of the finished parts was very much what was expected given the geometry of the mold. The double curved geometry as shown in Figure 13(c), which is actually a hemispherical shape in this research, was not absolutely achieved due to wrinkling of the reinforcement layers. This wrinkling can be reduced by tailored cutting of the reinforcement to adjust with the hemispherical shape but then there will be a major reduction of mechanical strength in the area where the reinforcement is cut.

Figure 14 shows the pictures of the closed loop composites where the first one is a hollow cylinder and the second one is an assembly of a flat cylinder web and a curved cylinder web. The shape conformance of the very complicated web was not as bad as expected but there is still room for improvement in the management of the VARTM process to better facilitate the manufacturing of the curved composites. Controlling the temperature and humidity will be very useful in case of environments with varying conditions.

\subsection{Overview of the Results}

The summary of tensile, shear and thickness results with standard deviations are shown in Table 3. This is actually a summary of the results shown in Figures 10-12. 
Table 3. Summary of physical testing results of the manufactured composites.

\begin{tabular}{cccc}
\hline Epoxy Glass Composite Type & $\begin{array}{c}\text { Tensile Strength } \\
(\mathrm{MPa})\end{array}$ & $\begin{array}{c}\text { Shear Strength } \\
(\mathrm{MPa})\end{array}$ & $\begin{array}{c}\text { Composite } \\
\text { Thickness }(\mathrm{mm})\end{array}$ \\
\hline Flat Single-Sided Mold & $200 \pm 4$ & $34 \pm 1$ & $2.9 \pm 0.1$ \\
Singly Curved Single-Sided Mold & $110 \pm 2$ & $18 \pm 0.2$ & $3.1 \pm 0.04$ \\
Singly Curved Double-Sided Mold & $104 \pm 5$ & $17 \pm 0.3$ & $2.8 \pm 0.08$ \\
Doubly Curved Single Sided Mold & - & $11 \pm 0.25$ & $4.4 \pm 0.06$ \\
Doubly Curved Double Sided Mold & - & $10 \pm 0.15$ & $3.5 \pm 0.08$ \\
Hollow Cylinder Closed Loop & - & $19 \pm 0.3$ & $3.1 \pm 0.1$ \\
Cylinder Web Flat & - & $13 \pm 0.3$ & $3.2 \pm 0.1$ \\
Cylinder Web Curved & - & $10 \pm 0.3$ & $3.4 \pm 0.1$ \\
\hline
\end{tabular}

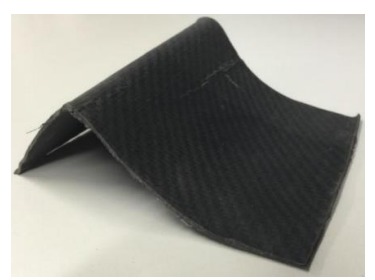

(a)

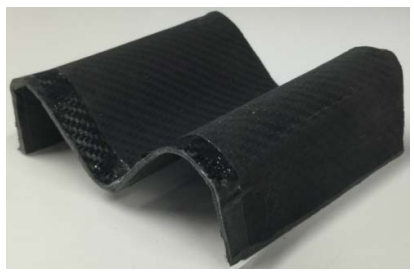

(b)

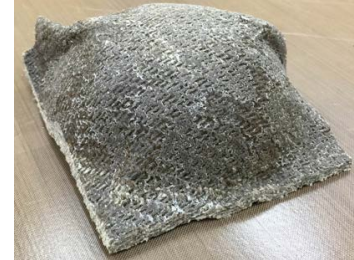

(c)

Figure 13. Pictures of (a) Simple curvature, (b) Singly curved and (c) Double curved composites.
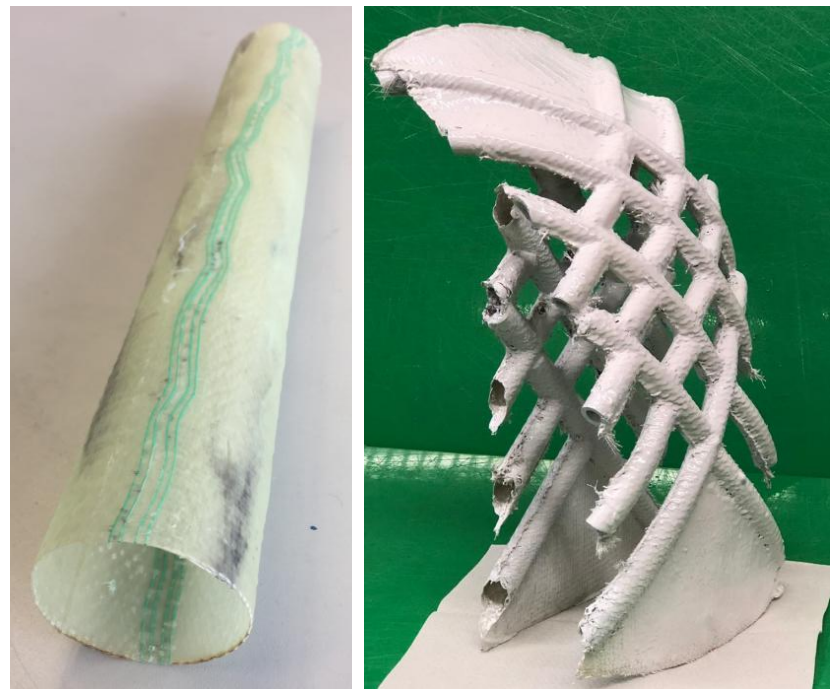

Figure 14. Pictures of hollow cylinder and hollow network flat and curved composite assembly.

\section{Conclusion}

This paper describes a method to manufacture curved composites using resin infusion (VARTM) and cheap PLA molds created by 3D printing (FDM). Three fiber reinforcements were used to successfully manufacture three types of curved composite profiles using single and double sided PLA molds. Shear strengths 
and thickness of each part were measured and the results were used to draw a few comparisons between the fiber types, mold types and the curvature profiles. The results showed that using double sided molds induces a better consistency in shape and dimensional accuracy of the composites compared to single sided molds. Shear strength decreases and thickness increases significantly from singly to doubly curved composite parts. A better composite quality can be achieved by modifying the basic resin infusion process (clamped inlet) but then again it will be a trade-off between the composite strength and the dimensional stability. It was found that the compaction pressure used in this research, which is almost equivalent to atmospheric pressure, supports the manufacture of the composites using the relatively weak PLA molds. Carbon fiber, glass fiber and flax, which are mostly used as reinforcements in the industry, were all supporting this method to produce curved epoxy resin composites. The tensile and shear strengths of the manufactured composites were mostly better or comparable to the ones in previous literature. This method will reduce the overall cost of manufacturing curved composites compared to processes using rigid molds especially in the case of one-off or low-scale productions. The manufactured profiles were conforming to the mold shape with a fair surface finish. A bridge structure containing a web of hollow cylinders was the most creative and complicated part of this project. Two types of bridges were manufactured (flat and curved). The curved bridge contained curved cylindrical webs that were curved in all three dimensions. Manufacturing of both these types required molding techniques which were more complicated than a standard VARTM manufacturing/molding technique. This research showed the capability of VARTM to produce epoxy composites with complicated profiles. This work can lead to the use of VARTM to manufacture closed-loop or hollow composites with better shape conformance and surface finish.

\section{Acknowledgements}

The Institut für Kunststoffverarbeitung Westpfalz (IKW).

\section{Conflicts of Interest}

The authors declare no conflicts of interest regarding the publication of this paper.

\section{References}

[1] Soutis, C. (2005) Fibre Reinforced Composites in Aircraft Construction. Progress in Aerospace Sciences, 41, 143-151. https://doi.org/10.1016/j.paerosci.2005.02.004

[2] Naser, M.Z. (2019) Extraterrestrial Construction Materials. Progress in Materials Science, 105, Article ID: 100577. https://doi.org/10.1016/j.pmatsci.2019.100577

[3] Kazmi, S., Govignon, Q. and Bickerton, S. (2019) Control of Laminate Quality for Parts Manufactured Using the Resin Infusion Process. Journal of Composite Materials, 53, 327-343. https://doi.org/10.1177/0021998318783308 
[4] Bellini, A., Guçeri, S.U. and Bertoldi, M. (2004) Liquefier Dynamics in Fused Deposition. Journal of Manufacturing Science and Engineering, 126, 237-246. https://doi.org/10.1115/1.1688377

[5] Zein, I., Hutmacher, D.W., Tan, K.C. and Teoh, S.H. (2002) Fused Deposition Modeling of Novel Scaffold Architectures for Tissue Engineering Applications. Biomaterials, 23, 1169-1185. https://doi.org/10.1016/S0142-9612(01)00232-0

[6] Ahn, S.-H., Montero, M., Odell, D., Roundy, S. and Wright, P.K. (2002) Anisotropic Material Properties of Fused Deposition Modeling ABS. Rapid Prototyping Journal, 8, 248-257. https://doi.org/10.1108/13552540210441166

[7] Montero, M., Roundy, S., Odell, D., Ahn, S.-H. and Wright, P.K. (2001) Material Characterization of Fused Deposition Modeling (FDM) ABS by Designed Experiments. Proceedings of Rapid Prototyping and Manufacturing Conference, Cincinnati, 15 May 2001, 10.

[8] Ang, K.C., Leong, K.F., Chua, C.K. and Chandrasekaran, M. (2006) Investigation of the Mechanical Properties and Porosity Relationships in Fused Deposition Modelling-Fabricated Porous Structures. Rapid Prototyping Journal, 12, 100-105. https://doi.org/10.1108/13552540610652447

[9] Anna, B. and Selçuk, G. (2003) Mechanical Characterization of Parts Fabricated Using Fused Deposition Modeling. Rapid Prototyping Journal, 9, 252-264. https://doi.org/10.1108/13552540310489631

[10] Bagsik, A. and Schöppner, V. (2011) Mechanical Properties of Fused Deposition Modeling Parts Manufactured with Ultem* 9085. ANTEC 2011, Boston, 1-5 May 2011, vol. 2, 1294-1298.

[11] Bagsik, A., Schoeppner, V. and Klemp, E. (2010) FDM Part Quality Manufactured with Ultem ${ }^{\star}$ 9085. 14th International Scientific Conference on Polymeric Materials, Halle (Saale), 15 September 2010, Vol. 15, 307-315.

[12] Sood, A.K., Ohdar, R.K. and Mahapatra, S.S. (2010) Parametric Appraisal of Mechanical Property of Fused Deposition Modelling Processed Parts. Materials \& Design, 31, 287-295. https://doi.org/10.1016/j.matdes.2009.06.016

[13] Sood, A.K., Chaturvedi, V., Datta, S. and Mahapatra, S.S. (2011) Optimization of Process Parameters in Fused Deposition Modeling Using Weighted Principal Component Analysis. Journal of Advanced Manufacturing Systems, 10, 241-259. https://doi.org/10.1142/S0219686711002181

[14] Gray, R.W., Baird, D.G. and Helge Bøhn, J. (1998) Effects of Processing Conditions on Short TLCP Fiber Reinforced FDM Parts. Rapid Prototyping Journal, 4, 14-25. https://doi.org/10.1108/13552549810197514

[15] Weinmann, J., Ip, H., Prigozhin, D., Escobar, E., Mendelson, M. and Noorani, R. (2003) Application of Design of Experiments (DOE) on the Processing of Rapid Prototyped Samples. The Solid Freeform Symposium, Proceedings, Austin, 2003, 340-347.

[16] Drummer, D., Cifuentes-Cuéllar, S. and Rietzel, D. (2012) Suitability of PLA/TCP for Fused Deposition Modeling. Rapid Prototyping Journal, 18, 500-507. https://doi.org/10.1108/13552541211272045

[17] Teoh, K.J. and Hsiao, K.-T. (2011) Improved Dimensional Infidelity of Curve-Shaped VARTM Composite Laminates Using a Multi-Stage Curing Technique-Experiments and Modeling. Composites Part A: Applied Science and Manufacturing, 42, 762-771. https://doi.org/10.1016/j.compositesa.2011.03.003

[18] Ho, M.-P., Wang, H., Lee, J.-H., Ho, C.-K., Lau, K.-T., Leng, J. and Hui, D. (2012) Critical Factors on Manufacturing Processes of Natural Fibre Composites. Compo- 
sites Part B: Engineering, 43, 3549-3562.

https://doi.org/10.1016/j.compositesb.2011.10.001

[19] Kuo, C.-C. (2012) A Simple and Cost-Effective Method for Fabricating Epoxy-Based Composite Mold Inserts. Materials and Manufacturing Processes, 27, 383-388. https://doi.org/10.1080/10426914.2011.551906

[20] Oterkus, E., Madenci, E., Weckner, O., Silling, S., Bogert, P. and Tessler, A. (2012) Combined Finite Element and Peridynamic Analyses for Predicting Failure in a Stiffened Composite Curved Panel with a Central Slot. Composite Structures, 94, 839-850. https://doi.org/10.1016/j.compstruct.2011.07.019

[21] Minakuchi, S., Umehara, T., Takagaki, K., Ito, Y. and Takeda, N. (2013) Life Cycle Monitoring and Advanced Quality Assurance of L-Shaped Composite Corner Part Using Embedded Fiber-Optic Sensor. Composites Part A: Applied Science and Manufacturing, 48, 153-161. https://doi.org/10.1016/j.compositesa.2013.01.009

[22] Kazmi, S., Jayaraman, K. and Das, R. (2016) Single-Step Manufacturing of Curved Polypropylene Composites Using a Unique Sheet Consolidation Method. Journal of Materials Processing Technology, 237, 96-112. https://doi.org/10.1016/j.jmatprotec.2016.05.028

[23] Zhao, Z., Jiang, D., Ou, Y., Tang, K., Luo, X. and Quan, Z. (2012) A Hollow Cylindrical Nano-Hydroxyapatite/Polyamide Composite Strut for Cervical Reconstruction after Cervical Corpectomy. Journal of Clinical Neuroscience, 19, 536-540. https://doi.org/10.1016/j.jocn.2011.05.043

[24] Kim, P.J., Lee, D.G. and Choi, J.K. (2000) Grinding Characteristics of Carbon Fiber Epoxy Composite Hollow Shafts. Journal of Composite Materials, 34, 2016-2035. https://doi.org/10.1177/002199800772661958

[25] Gordon, J. and Jeronimidis, G. (1980) Composites with High Work of Fracture. Philosophical Transactions of the Royal Society of London A, 294, 545-550. https://doi.org/10.1098/rsta.1980.0063

[26] Summerscales, J. (2010) A Taxonomy for Resin Infusion Processes.

[27] Schuster, J., Kazmi, S.M.R. and Lutz, J. (2015) Manufacturing and Testing of Curved Fibrecomposites Using Vacuum Assisted Resin Transfer Moulding (VARTM). 20th International Conference on Composite Materials (ICCM-20), Copenhagen, 19-24 July 2015.

[28] Schuster, J., Govignon, Q. and Bickerton, S. (2014) Processability of Biobased Thermoset Resins and Flax Fibres Reinforcements Using Vacuum Assisted Resin Transfer Moulding. https://doi.org/10.4236/ojcm.2014.41001

[29] Dong, C. (2008) A Modified Rule of Mixture for the Vacuum-Assisted Resin Transfer Moulding Process Simulation. Composites Science and Technology, 68, 2125-2133. https://doi.org/10.1016/j.compscitech.2008.03.019

[30] Kazmi, S., Das, R. and Jayaraman, K. (2014) Sheet Forming of Flax Reinforced Polypropylene Composites Using Vacuum Assisted Oven Consolidation (VAOC). Journal of Materials Processing Technology, 214, 2375-2386.

https://doi.org/10.1016/j.jmatprotec.2014.04.030

[31] Summerscales, J. (2011) Resin Infusion under Flexible Tooling (RIFT). In: Wiley Encyclopedia of Composites, Wiley, Hoboken, 1-11. https://doi.org/10.1002/9781118097298.weoc216

[32] Zhu, Q., Geubelle, P.H., Li, M. and Tucker III, C.L. (2001) Dimensional Accuracy of Thermoset Composites: Simulation of Process-Induced Residual Stresses. Journal of Composite Materials, 35, 2171-2205. https://doi.org/10.1177/002199801772662000 
[33] Bogetti, T.A. and Gillespie Jr., J.W. (1992) Influence of Cure Shrinkage on Process-Induced Stress and Deformation in Thick Thermosetting Composites. Army Ballistic Research Lab Aberdeen Proving Ground Md.

[34] Miller, K. and Ramani, K. (1999) Process-Induced Residual Stresses in Compression Molded UHMWPE. Polymer Engineering \& Science, 39, 110-118. https://doi.org/10.1002/pen.11401

[35] Ruiz, E. and Trochu, F. (2005) Numerical Analysis of Cure Temperature and Internal Stresses in Thin and Thick RTM Parts. Composites Part A: Applied Science and Manufacturing, 36, 806-826. https://doi.org/10.1016/j.compositesa.2004.10.021

[36] Golestanian, H. and El-Gizawy, A.S. (2001) Modeling of Process Induced Residual Stresses in Resin Transfer Molded Composites with Woven Fiber Mats. Journal of Composite Materials, 35, 1513-1528.

https://doi.org/10.1106/VW5C-GN89-UXKR-WFKT

[37] Fan, Z., Santare, M.H. and Advani, S.G. (2008) Interlaminar Shear Strength of Glass Fiber Reinforced Epoxy Composites Enhanced with Multi-Walled Carbon Nanotubes. Composites Part A: Applied Science and Manufacturing, 39, 540-554.

https://doi.org/10.1016/j.compositesa.2007.11.013

[38] Yang, B., Kozey, V., Adanur, S. and Kumar, S. (2000) Bending, Compression, and Shear Behavior of Woven Glass Fiber-Epoxy Composites. Composites Part B: Engineering, 31, 715-721. https://doi.org/10.1016/S1359-8368(99)00052-9

[39] Almeida Jr., J.H.S., Angrizani, C.C., Botelho, E.C. and Amico, S.C. (2015) Effect of Fiber Orientation on the Shear Behavior of Glass Fiber/Epoxy Composites. Materials \& Design (1980-2015), 65, 789-795.

https://doi.org/10.1016/j.matdes.2014.10.003

[40] Selmy, A., Elsesi, A., Azab, N. and El-baky, M.A. (2012) Interlaminar Shear Behavior of Unidirectional Glass Fiber (U)/Random Glass Fiber (R)/Epoxy Hybrid and Non-Hybrid Composite Laminates. Composites Part B: Engineering, 43, 1714-1719. https://doi.org/10.1016/j.compositesb.2012.01.031

[41] Godara, A., Mezzo, L., Luizi, F., Warrier, A., Lomov, S.V., Van Vuure, A.W., Gorbatikh, L., Moldenaers, P. and Verpoest, I. (2009) Influence of Carbon Nanotube Reinforcement on the Processing and the Mechanical Behaviour of Carbon Fiber/Epoxy Composites. Carbon, 47, 2914-2923.

https://doi.org/10.1016/j.carbon.2009.06.039

[42] Yan, L. and Chouw, N. (2015) Effect of Water, Seawater and Alkaline Solution Ageing on Mechanical Properties of Flax Fabric/Epoxy Composites Used for Civil Engineering Applications. Construction and Building Materials, 99, 118-127. https://doi.org/10.1016/j.conbuildmat.2015.09.025

[43] Devendra, K. and Rangaswamy, T. (2013) Strength Characterization of E-Glass Fiber Reinforced Epoxy Composites with Filler Materials. Journal of Minerals and Materials Characterization and Engineering, 1, 353-357. https://doi.org/10.4236/jmmce.2013.16054 